Document downloaded from:

http://hdl.handle.net/10251/52190

This paper must be cited as:

Andrés, B.; Poler Escoto, R. (2013). A Method to Quantify the Power Distribution in Collaborative Non-hierarchical Networks. IFIP Advances in information and communication technology. 408:660-669. doi:10.1007/978-3-642-40543-3.

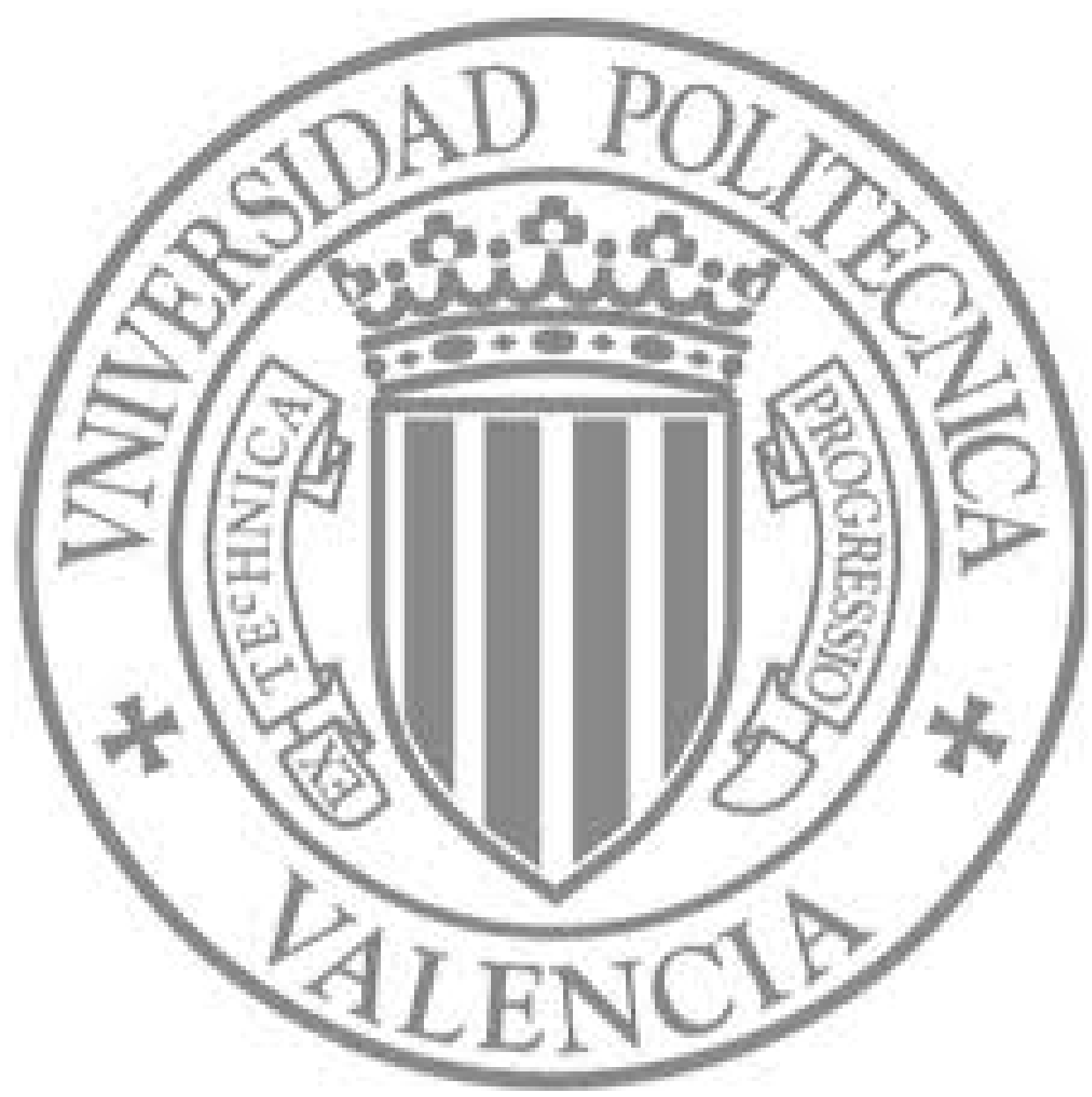

The final publication is available at

http://www.springerprofessional.de/069---a-method-to-quantify-the-power-distribution-in-colle Copyright Springer Verlag (Germany) 


\title{
A method to quantify the power distribution in collaborative non-hierarchical networks
}

\author{
Beatriz Andrés ${ }^{1}$, Raúl Poler ${ }^{1}$, \\ ${ }^{1}$ Research Centre on Production Management and Engineering (CIGIP), \\ Universitat Politècnica de València (UPV). Plaza Ferrándiz y Carbonell, 2, 03801 Alcoy, Spain \\ \{beaanna, rpoler\}@cigip.upv.es
}

\begin{abstract}
Collaborative networks are characterised by the establishment of relations in more or less hierarchical power structures. The hierarchy of the network is defined by the partners' power degree. Hierarchical structures and associated barriers limit the decision making and discourage collaboration within partners. This paper focuses on proposing a method to allow researchers to identify the power degree of each network partner, through Markov Chains. Knowing the power distribution, helps researchers to diagnose the power balance, reconsider the status in the network and have a better view of power interaction and collaboration. Therefore, the power distribution analysis is a key issue to understand the partners' behaviour and achieve sustainable networks.
\end{abstract}

Keywords: networks, power distribution, Markov Chain, sustainable network

\section{Introduction}

Research in collaborative networks has increased nowadays due to the companies' participation in this networks leads them to achieve inherent collaborative advantages [1]. Collaboration within a network can be centralised or decentralised. Centralised approaches, [2] in which a single decision unit possesses all the power, are associated with the concept of hierarchical networks (HN) where dominant nodes possess the power and the secondary ones must adapt [3]. In today's dynamic environment, it is important to consider the objectives of both dominant and secondary partners. In light of this, decentralised approaches are considered; in this case non-hierarchical networks (NHN) are identified, in which power distribution is balanced, in principle, and all the partners are equally considered [3]. Nevertheless, the power asymmetry among NHN partners is evidenced [4] as a result of the information restriction, the task dependency, the roles in the network, the activities in the product development, etc. [5]. Thus, the power of each member of the network influences the partners' decisions, making interesting the study of the power distribution in order to promote more balanced networks. The way how industries behave affects their survival in the current environment, characterised by the economic crisis. Besides considering all the alternatives to be applied within the enterprises, researchers have to also consider the inter-enterprise relationships established in the network. In order to deal with the economic issue in a way, the network redesign will allow researchers to achieve 
power balanced structures; therefore, enterprises will be reinforced and will obtain offset gains allowing them to reinvigorate the economy. Network sustainability issues are clearly in the pole position of the topic under study due to the power distribution analysis implies to build balanced collaborative relationships and achieve value chain networks. For these reasons, the power distribution in collaborative networks needs to be studied in order to achieve and develop a well-integrated network.

This paper particularly focuses on collaborative and non-hierarchical networks due to the emerging importance for researchers [3]; nevertheless, the developed method can be applied for the wide variety of network topologies. Modelling and analysing the individual power will allow researchers to identify or predict conflicts, select potential members that best fit in the network and establish the correct assignment of partners. The main aim of this paper is to propose a method to help researchers on the identification of the relations established within network members with the main aim of quantifying the power distribution in the network.

\section{Problem definition}

The common understanding of the power concept is similar to authority. Nevertheless, the power notion is far from a simple phenomenon and it is not simply related with the size of the companies. Different authors such as [6] and [7] develop methodologies to analyse the partners' power. Moreover, Liu and Zolghadri [4] focus on the power evaluation according to the partners' activities and involvement in the product development.

This paper goes further and provides a general method to compute the power distribution within the network not only considering variables related with the product, but also considering a wide variety of measures such as contracts established, products exchanged, monetary transactions, etc.

In order to develop the paper, two questions are raised: (i) what are the measures used when defining the power distribution and (ii) how to use these measures in order to compute the power distribution in a network. In light of this, this paper is focused on the definition a set of measures (section 3) and demonstrate how these measures can be properly used to calculate the transition probabilities in order to apply the Markov chains analysis and estimate the network power distribution (section 4). Hence, in networks where there is not a clear leader, researchers can analyse the power distribution to identify those partners with a higher power, with the main aim of balancing the authority.

\section{Measures to identify the power distribution}

A set of measures considering more than one perspective are given in this section to compute the partners' relationships and quantify the power distribution within the network. The measures are provided taking into account that the method to quantify the power distribution is based on Markov Chain analysis. The proposed measures are set out to identify relationships between nodes. Linkages between partners can be 
identified through a graph representing pair wise relationships, using nodes symbolising the partners, and arrows symbolising linkages between them (figure 1).

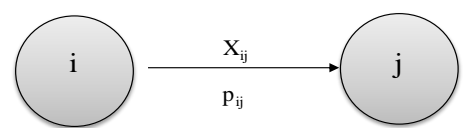

$X_{i j}$ : quantifiable measure to identify the relationship between node $i$ and its partner in the network $j$. Power Measurement $\mathrm{p}_{\mathrm{ij}}$ : probability of moving from state $i$ to state $j$, this probability is used to analyse the power through Markov Chains

Figure 1. Transaction diagram simplified for two network nodes

Due to the nature of the developed method, based on Markov chains, the measures to compute the power within a network are leaded to relate the transactions established between pairs of network nodes $\left(\mathrm{p}_{\mathrm{ij}}\right)$. Table 1 defines and describes the measures to compute the power and relates these measures with the partners' power (PW) considering that the higher $(\uparrow)$ or lower $(\downarrow)$ value of $p_{i j}$ implies a higher $(\uparrow)$ or lower $(\downarrow)$ power of the node to which the transaction is assigned.

Table 1. Measures to compute the network power distribution $\left(X_{i j}\right)$

\begin{tabular}{|c|c|c|c|}
\hline Measure & Description & $p_{i j}$ & $P W$ \\
\hline Contracts & $\begin{array}{l}\text { Number of contracts established between node } i \text { and node } j . \text { The greater number of contracts accepts } \\
\text { the node } \mathrm{i} \text { with the node } \mathrm{j} \text { the greater power will have } \mathrm{j} \text { over } i\end{array}$ & $\uparrow$ & $\uparrow$ \\
\hline End Product & $\begin{array}{l}\text { Participation of the products developed at node } i \text { on product performed by } j \text {. The importance degree } \\
\text { is associated to the partner who send products more significant for the end product, node } i\end{array}$ & $\uparrow$ & $\uparrow$ \\
\hline $\begin{array}{l}\text { Exchange of } \\
\text { Information }\end{array}$ & $\begin{array}{l}\text { Information exchanged between nodes. The most important node is one that receives more } \\
\text { information from the other nodes }\end{array}$ & $\uparrow$ & $\uparrow$ \\
\hline Frequency & $\begin{array}{l}\text { Regarding the payment, exchange of information, products shipped. The powerful node is one that } \\
\text { establishes transactions in lower times. That is where } \mathrm{p}_{\mathrm{ij}} \text { is lower }\end{array}$ & $\downarrow$ & $\uparrow$ \\
\hline Innovation & $\begin{array}{l}\text { Percentage of patents sold to each partner. The powerful partner buys more patents. The measure } \\
\text { can also be measured in terms of the economic value of patents }\end{array}$ & $\uparrow$ & $\uparrow$ \\
\hline $\begin{array}{l}\text { Monetary } \\
\text { Units }\end{array}$ & $\begin{array}{l}\text { Based on the transaction of monetary units between nodes. Incomes. The higher profits has the } \\
\text { node, the more powerful is considered }\end{array}$ & $\uparrow$ & $\uparrow$ \\
\hline $\begin{array}{l}\text { Material } \\
\text { transactions }\end{array}$ & $\begin{array}{l}\text { Number of products transferred between partners. The greater amount of products shipped to one } \\
\text { partner, the more powerful is considered the node that receives the products }\end{array}$ & $\uparrow$ & $\uparrow$ \\
\hline \multirow[t]{2}{*}{ Orders } & $\begin{array}{l}\text { Number of products ordered, order volume, product delays, etc. Average delays to each customer } \\
\text { could be an applicable approach to describe the orders measure. The less average delays has one } \\
\text { node, the more powerful }\end{array}$ & $\downarrow$ & $\uparrow$ \\
\hline & nvolves the workload. The more orders made the more powerful is the node & $\uparrow$ & $\uparrow$ \\
\hline Partners & $\begin{array}{l}\text { ablished between the network partners. The greater number of } \\
\text { node, the most important is the node }\end{array}$ & $\uparrow$ & $\uparrow$ \\
\hline $\begin{array}{l}\text { Product } \\
\text { Value }\end{array}$ & $\begin{array}{l}\text { VAT or benefits obtained by the network node. The higher profits the node has, the more powerful } \\
\text { is. Benefits }=\text { monetary units from node } j \text { to node } i \text { - monetary units spent to manufacture the } \\
\text { product in node } i\end{array}$ & $\uparrow$ & $\uparrow$ \\
\hline \multirow[b]{2}{*}{ Products } & Total sales as monetary transactions (Price). The higher sales receives a node, the more powerful is & $\uparrow$ & $\uparrow \uparrow$ \\
\hline & $\begin{array}{l}\text { Total sales as products transferred from one node to another. The higher products transfer the more } \\
\text { powerful is the node }\end{array}$ & $\uparrow$ & $\uparrow$ \\
\hline \multirow[t]{2}{*}{ Purchase } & $\begin{array}{l}\text { Number of purchased products. Returned products: number of products returned from the customer } \\
\text { node to the supplier. The more products returned the more powerful is the node that returns a } \\
\text { product }\end{array}$ & $\uparrow$ & $\uparrow$ \\
\hline & $\begin{array}{l}\text { Cost of purchase: monetary units associated with the purchase, material costs, administration costs, } \\
\text { etc. Swithching costs: monetary units refunded to the customer due to the product has been returned }\end{array}$ & $\uparrow$ & $\uparrow$ \\
\hline
\end{tabular}
$p_{i j}$ : transaction probability form $i$ to $j / P W$ : power.

This paper is bound to generate a methodology for the identification of the power distribution within the network partners. Therefore, it is out of the scope the definition of the method to obtain and calculate $X_{i j}$ and $p_{i j}$ variables. Nevertheless, for better 
understanding, if the researcher decides to compute the power considering the monetary units measure, $\mathrm{X}_{\mathrm{ij}}$ is defined by the number or value of the monetary transactions between the partner $i$ and the partner $j$. The transaction probability is obtained as regards the $X_{\mathrm{ij}}$ transactions, considering the percentage of the monetary units sent from node $i$ to $j$ over the total monetary units sent by $i$ to all the nodes in which establish transactions $\rightarrow p_{i j}=X_{i j} / \sum X_{i j}$. In this case, the higher $p_{i j}$ means the higher number of monetary units transacted from the node $i$ to the $j$, so that, the node $j$ will be the more powerful as regards $i$.

All the measures should be treated with equally importance. The selection of one or another measure will depend on the network, the available data, or even the way the researcher wants to estimate the power distribution. It is difficult to calculate the transition probabilities, however, there can be known through surveys distributed to the networked partners, giving the researcher an insight of the relationships established between the nodes; or just asking partners for data of interest to calculate the distribution of power.

Employing Markov chains for the power distribution implies to relate the powerful node with that one receiving many links (transactions) from the rest of network nodes. Thus, the more links the node has associated, the more important will be due to the measure transactions are defined considering the relations established within the network members.

\section{Method to quantify the power: Markov Chain Model}

Markov chains (MC) analysis provides information about the changes taking place in a system and the probability that at any future time the system will be in a particular state. In network contexts, Markov Chains Models (MCM) are used to model the partners' retention and partners' migration situations regarding the relations they establish [8-9]; being suitable to model and estimate the power within the network. For this propose, this paper is developed to measure the relationships and links between nodes and determine where the transactions lead in order to identify the power distribution. The network is considered the system $(S)$ and the nodes $(i)$ represent the states in the Markov chain analysis. Transactions between nodes are met at most once a period. A key feature of the nodes' transactions is that the future prospects for the nodes relationship are a function only of the current state of the relationship and not of the particular path of transactions took to reach the current state; this property is called the Markov property [10-11]. Markov analysis describes the system transition towards a certain state, through probabilities, named transition probabilities $p_{i j}$, that is $\operatorname{Pr}\left(X_{t+1}=j \mid X_{t}=i\right)=p_{i j}(n)$; where, $p_{i j}{ }^{(n)} \geq 0$ for all pairs $i, j \in S$ and $\sum_{j} \in_{S} p_{i j}^{\left({ }^{(n)}\right.}=1$. Transition probabilities of a stationary MC can be arranged in the transition matrix. Furthermore, the transition matrix measures can be represented in an associated graph whose vertex represent the states from the system $S$ and between the vertex $i$ and vertex $j$ an arrow $(i, j)$ is represented, if and only if $p_{i j}>0$.

The steady-state is one of the Markov chains properties used to determine the balance of power among partners. The Markovian property indicates that in the long term the process is stabilised and the operating characteristics of the system become 
time independent. The steady state probabilities are noted as $\pi_{j}$ and there are characterised by $\lim _{n \rightarrow \infty} p_{i j}^{(n)}=\pi_{j}$ where $\pi_{j}$ satisfy the following equations:

(i) $\pi_{j}>0$

(ii) $\pi_{j}=\sum_{i=0}^{M} \pi_{i} * p_{i j}, \mathrm{j}=0,1, \ldots \mathrm{M}$

(iii) $\sum_{j=0}^{M} \pi_{j}=1$.

Among the states some are transient and the others absorbing. If the network has some absorbing states the chain is called absorbing chain [12]. An absorbing state " $i$ " is one that endures; that is, starting from any of the transition states the process terminates once it reaches the absorbing state $\left(p_{i i}=1\right)$ [10]. For Markov chains analysis absorbing states are not considered implying that all the steady state probabilities are zero $\Pi_{j^{*} i}=0$ except those of the absorbing states, $\Pi_{i}=1$, being the results inconclusive to study any future behaviour of the Markov chain states. Different authors treat the Markov Chain Problem with an Absorbing State [8, 10, 11, 12, 13]. In this paper the absorbing state is treated through dummy players and fictitious transactions.

The method used to compute the power distribution within a network is summarised as follows in seven steps.

STEP 1. Once the network is identified and modelled, the researcher proceeds to select the measures (from table 1) to be used in the identification of relations within pair wise nodes. The measures are selected according to the researchers' requirements to calculate the power distribution within the network.

STEP 2. Depending on the measure selected to compute the power distribution, the transactions flow in one direction or another. Two examples are the material transactions and the monetary units. The first measure produces a flow from the supplier to the customer, whilst in the second the flow is generated from the customer to the suppler. For the network and transactions representation the identification of the flow direction within the network, is to be considered due to depends on the selected measure.

STEP 3. Define the initial transaction matrix $(P)$ that consists on the transitions established among all the networked nodes. The transition probabilities are calculated through the equations provided in section 3. The use of Markov chains to compute the power distributions is limited by the appearance of the absorbing states. The initial transition matrix $(\mathrm{P})$ is characterised by having absorbing states. The absorbing state location depends on the flow direction, directly influenced by the type of measure.

STEP 4. The absorbing states are to be removed through the insertion of a dummy node(s) and the establishment of a fictitious transaction(s) within the network nodes. In network systems the network end nodes are considered as absorbing as all the arrows are headed to these end nodes such as customer or raw material suppliers. The fact that an absorbing state is one node or another depends on the flow direction. Since one node does not generate any transaction to any other node, then this node is the absorbing state and it is mathematically represented by $p_{i i}=1$. Absorbing states must not be considered in the transition matrix. The introduction of a dummy node and a fictitious transaction does not affect the system and helps researchers to remove the absorbing states in order to calculate the transition probabilities of the modeled network and consequently compute the power distribution.

STEP 5. Final transition matrix $\left(P^{\prime}\right)$ with no absorbing states. Markov chains do not admit absorbing states due to this does not make sense to calculate transition 
probabilities. Therefore, once the dummy nodes and transactions are introduced in the network in order to remove the absorbing states, the new network is modelled. The new network with dummy nodes and transition probabilities is now modelled from the results of the final transition matrix ( $\left.\mathrm{P}^{\prime}\right)$.

STEP 6. Steady state probabilities $\left(\Pi_{i}\right)$ calculation for all the nodes including the dummy players, introduced in the network to remove the absorbing states. Once the transition matrix has no absorbing states, the probabilities are calculated in the long term in order to achieve the steady-state probabilities (equation 1). These probabilities will be conditioned by the results of the dummy nodes. This means that, the calculated steady-state probabilities of all the nodes located after or before the dummy node are also considered in the calculation of the steady state probabilities.

STEP 7. Steady state probabilities normalisation $\left(\Pi_{i}{ }^{\prime}\right)$, by only considering the networked nodes, that is without considering the dummy nodes. The consideration of the dummy nodes in the calculation of the steady state probabilities $\left(\Pi_{i}\right)$ gives us a distorted view of the real distribution of the power. Thus, the steady state probabilities are to be recalculated, regardless the steady state probabilities of the dummy nodes. The recalculation leads to compute the normalised steady-state probabilities $\left(\Pi_{i}{ }^{\prime}\right)$ through the equation provided in table 2 .

Table 2. Steady state probabilities normalisation

\begin{tabular}{|c|c|}
\hline$\Pi_{i}^{\prime}=\frac{\Pi_{i}}{{ }^{\prime}}$ & $\sum \Pi_{j}$ ' the sum of the steady-state probabilities excluding the steady-state probabilities of dummy nodes \\
\hline$\Pi_{i}-\overline{\sum \Pi_{j}^{\prime}}$ & $\Pi_{i}$ the steady state probability of node $i$, considering the whole system, including dummy nodes \\
\hline & $\begin{array}{l}\boldsymbol{\Pi}_{i} \text { ' normalised steady-state probability of the node } i \text { considering only the nodes of the network without } \\
\text { taking into account the steady state probabilities of the dummy nodes }\end{array}$ \\
\hline
\end{tabular}

In our case, the system is represented by a network with different nodes. The provided method allows researchers to determine how the network evolves in order to identify those members with higher power proportion.

\subsection{Numerical Example}

The following example illustrates the notion that researchers can use MCM not only to evaluate the power distribution, but also to manage and improve networked nodes relationships and therefore balance the power in the network in order to make it more sustainable. Using Markov chains allows researchers to estimate the power distribution at the long term within a network considering both the transition probabilities and the steady-state probabilities.

Namely, for the development of our paper, we propose a numerical example. The methodology is in the early stages of development and therefore has not yet reached the stage of implementation in a real network. However we are working on the search of a network in order to apply the methodology in a more complex network environment that would give researchers a better insight of the methodology strength. In the example hereafter developed it is considered the measure of monetary units' transaction so that the transaction flow is established from the customer to the supplier. We construct a Markov chain with 11 transient states (networked nodes) 
$\left\{\mathrm{A}_{2}, \mathrm{~A}_{3}, \mathrm{~A}_{4}, \ldots, \mathrm{A}_{12}\right\}$. The absorbing states depicted correspond to the network suppliers $\left\{\mathrm{A}_{2}, \mathrm{~A}_{3}\right\}$ where, $p_{22}=1$ and $p_{33}=1$.

The transition probability matrix is generated as a result of the transition probabilities of the monetary transactions among the different nodes (figure 2). The model may also be represented as a graph as shown in figure 3 a.

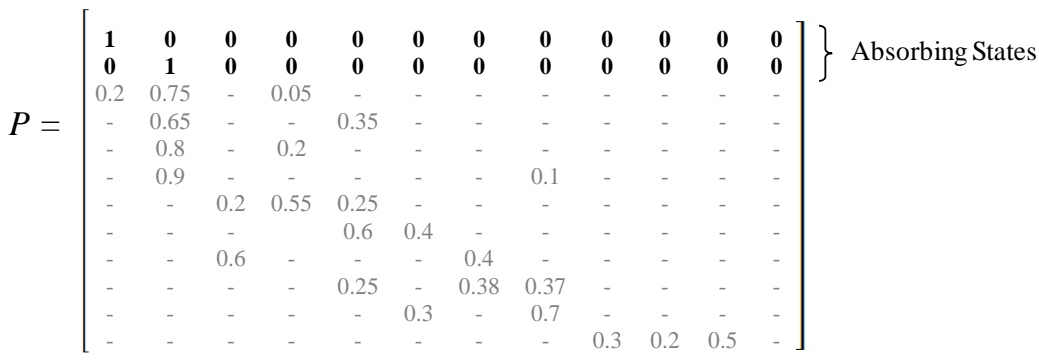

Figure 2. Probability transaction matrix of monetary units' transaction

In order to remove the absorbing states, a dummy node, the raw materials supplier $\left\{\mathrm{A}_{1}\right\}$ is depicted; moreover, the customer is now considered a dummy node $\left\{\mathrm{A}_{\mathrm{k}}\right\}$ due to the power distribution is to be calculated by only considering the proper network nodes, that is, suppliers, manufacturers, assemblers and warehouses. An arrow from the raw materials supplier node is generated, in the form of monetary units' transaction, towards the customer to set a fictitious transaction that can be represented by taxes to the society. Thus, considering the new dummy nodes and the fictitious transaction the new graph is outlined in figure 3(b).

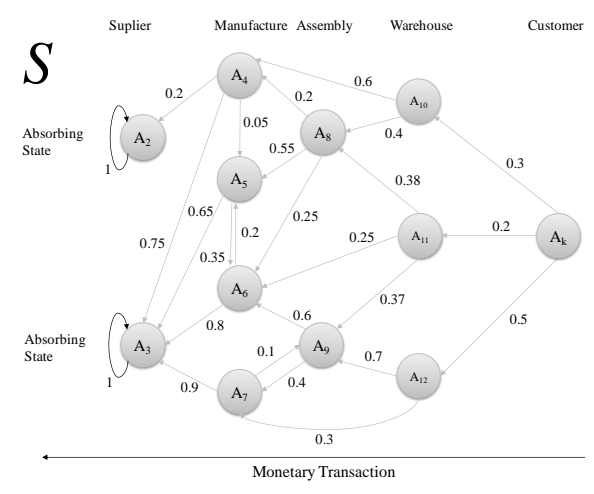

(a) Numerical Example - Considering absorbing states

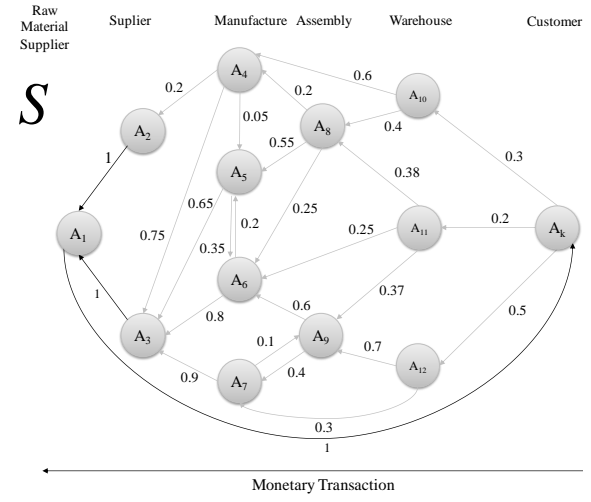

(b) Numerical Example - With none absorbing states

Figure 3. Associated graph

A new transition matrix is represented considering no-absorbing states and considering the raw materials supplier dummy node and the fictitious transaction from the raw materials supplier to the customer node (figure 4). 


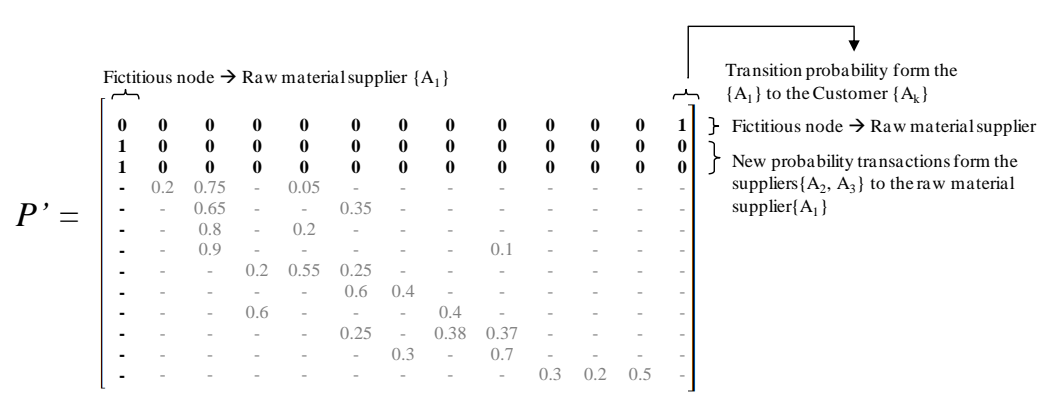

Figure 4. Probability transition matrix of monetary units with none absorbing states

A matrix with no-absorbing states has been generated $\left(p_{i i} \neq 1\right)$. Thus we proceed to calculate the steady-state probabilities $\left(\pi_{i}\right)$ that will allow researchers to estimate the power distribution. Concretely, the transition matrix is set for the transition probabilities considering the exchange of monetary units in a one year period. Thus, the steady-state probabilities are generated to identify the most powerful nodes in the long term, based on the monetary units' transactions.

The steady state probabilities are first calculated considering all the nodes, including the dummy nodes raw materials supplier and customer (equation 1). The results of the steady-state probabilities for the whole network are depicted in table 3 in the row represented by $\pi_{i}$. As aforementioned and given that the dummy nodes, raw materials supplier and customer, are not to be considered in the power distribution estimation (figure 5), the equation (2) provided in table 2 is to be applied in order to normalise the results of the steady-state probabilities taking into account only the steady-state probabilities of the suppliers, manufacturers, assemblers and warehouses nodes $\left\{\mathrm{A}_{2}, \mathrm{~A}_{3}, \mathrm{~A}_{4}, \ldots, \mathrm{A}_{12}\right\}$. Thus, the normalised steady state probabilities are depicted in the second row of table 3 , represented by $\pi_{i}$.

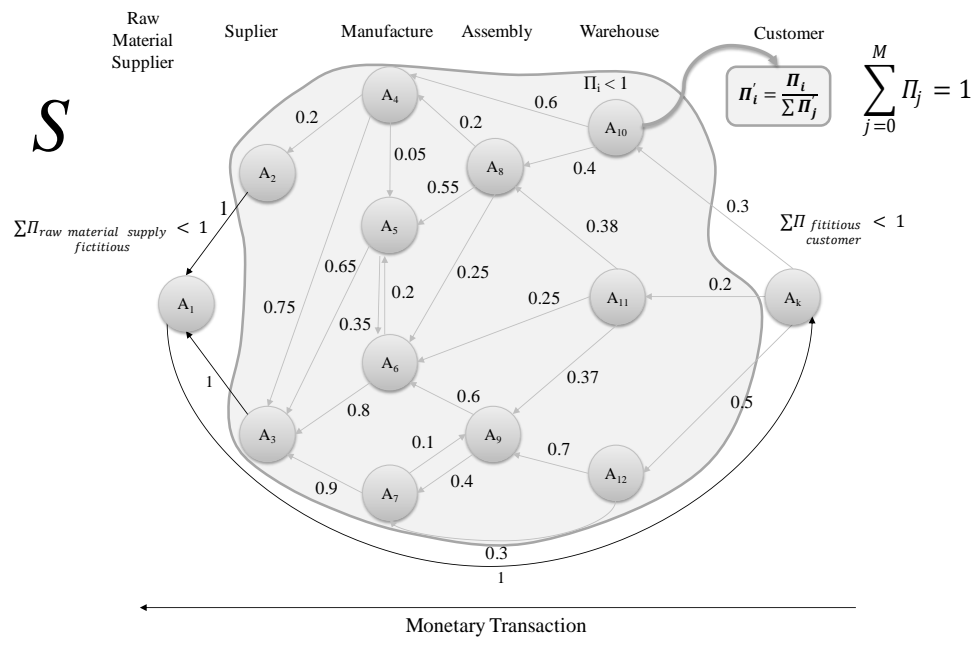

Figure 5. Normalised steady state probabilities (dummy nodes are not considered) 
Table 3. Steady state probabilities

\begin{tabular}{l|c|c|c|c|c|c|c|c|c|c|c|c|c|c}
\hline Nodes & $\mathbf{A}_{\mathbf{1}}$ & $\mathbf{A}_{\mathbf{2}}$ & $\mathbf{A}_{\mathbf{3}}$ & $\mathbf{A}_{\mathbf{4}}$ & $\mathbf{A}_{\mathbf{5}}$ & $\mathbf{A}_{\mathbf{6}}$ & $\mathbf{A}_{\mathbf{7}}$ & $\mathbf{A}_{\mathbf{8}}$ & $\mathbf{A}_{\mathbf{9}}$ & $\mathbf{A}_{\mathbf{1 0}}$ & $\mathbf{A}_{\mathbf{1 1}}$ & $\mathbf{A}_{\mathbf{1 2}}$ & $\mathbf{A}_{\mathbf{k}}$ & $\mathbf{A V G}\left(\boldsymbol{\pi}_{\mathbf{i}} \mathbf{)}\right.$ \\
\hline $\boldsymbol{\pi}_{\mathbf{i}}$ & 0,1707 & 0,0075 & 0,1632 & 0,0374 & 0,0355 & 0,0761 & 0,0568 & 0,0334 & 0,0780 & 0,0512 & 0,0341 & 0,0853 & 0,1707 & \\
\hline $\boldsymbol{\pi}_{\mathbf{i}}{ }^{\prime}$ & & 0,0114 & $\mathbf{0 , 2 4 7 7}$ & 0,0568 & 0,0539 & $\mathbf{0 , 1 1 5 6}$ & 0,0863 & 0,0508 & $\mathbf{0 , 1 1 8 5}$ & 0,0777 & 0,0518 & $\mathbf{0 , 1 2 9 5}$ & & 0,0909 \\
\hline
\end{tabular}

Amongst all the steady-state probabilities already normalised $\left(\pi_{i}^{\prime}\right)$, we now proceed to determine which node is more powerful. In order to do that, the average of the normalised steady-state probabilities is calculated regarding equation (3).

$A V G\left(\pi_{i}{ }^{\prime}\right)=\frac{\sum_{i=2}^{m=k-1} \pi_{i}{ }^{\prime}}{\text { Nnodes }- \text { Ndummy_nodes }} \quad$ being $\mathrm{k}=$ the number of nodes

Those nodes with normalised steady state probabilities $\left(\pi_{i}^{\prime}\right)$ higher than normalised state probabilities average $\left(A V G\left(\pi_{i}\right)\right)$ are considered to be more powerful nodes. Specifically, in the numerical example the threshold valued regarding the average is $A V G\left(\pi_{i}\right)=0,09091$. In our particular case, $\left\{A_{3}, A_{6}, A_{9}, A_{12}\right\}$ nodes have greater power within the network. The most powerful nodes of the network, that hinder the network balance in terms of power is concerned, are identified on the basis that the power has been established based on monetary unit transactions.

Most of the networks are controlled by one or two dominant nodes, in that case, the network would be balanced regarding this one or two nodes. Nevertheless, if the network consists of six nodes, four of them more powerful, all the network will be balanced. The way of balancing the power will depend on the results obtained from the methodology application proposed in this paper. Therefore, the balance will be devoted to balance each network node or the one or two more powerful ones.

So that, whether the power accumulation worsens the network operation, researchers must propose a reduction of the power imbalance. Once the researcher has an insight of the power distribution within the network, decisions and actions are led to consider the entry of new nodes or reduce the powerful ones in order to enhance the power balance. Definitely, decisions related with the network redesign and network decision making system to apply decentralised and collaborative relationships, the network integration and the partners' alignment and selection are settled out. The information derived from the methodology provided allows researchers to improve the relationship among the network partners and achieve more sustainable networks.

\section{Conclusions}

Non-hierarchical networks are considered to be power balanced, nevertheless real networks are far to be balanced, due to the different activities and roles of the networked partners influencing the decision making. This makes interesting the study of power distribution within the network. Therefore, a method with seven steps has been developed to help researchers to estimate the power distribution within a 
network. The method uses the Markov Chain analysis in order to consider the power distribution in the long term. An example has been provided for better understanding.

Regarding the results derived from the method application, it can be concluded that the provided methodology is a useful tool, for researchers, to establish decisions regarding the identification of new and more appropriate networked partners in order to achieve an optimum network design. A significant outcome of this work is that researchers can use the results to achieve balanced and sustainable networks through selecting the most suitable and power balanced partners. As future research lines, the method is to be applied in a real network, that will give researchers an insight of how useful can be the method and also to improve the weakness parts of it.

\section{References}

1. Poler, R..: Intelligent Non-Hierarchical Manufacturing Networks (iNet-IMS). Intelligent Manufacturing Systems. Obtained from http://www.ims.org/sites/default/files/iNetIMS\%20MTP\%20Initiative\%202009\%20v1.3.doc (2010)

2. Schneeweiss, C.: Distributed decision making - a unified approach. European Journal of Operational Research, 150, pp. 23--252 (2003)

3. Andrés, B., Poler, R.: Relevant Problems in Collaborative Processes of Non-Hierarchical Manufacturing Networks. In Prado JC, García J, Comesaña JA, Fernández AJ (eds.), 6th International Conference on Industrial Engineering and Industrial Management, pp. 90-97,Vigo (2012)

4. Liu, Y., Zolghadri, M.: Power Interaction in Non-hierarchical Supply Chain Network. Proceedings of the 2011 17th International Conference on Concurrent Enterprising, pp. 1$-8(2011)$

5. CONVERGE: Work Package Name: System Requirements \& Reference Model DefinitionCollaborative Communication Driven Decision Management in Nonhierarchical Supply Chains of the Electronic Industry. D2.2 Decision-Making Model, Data Mapping and Integration Roadmap (2010)

6. Emerson, R.M.: Power-dependence relations. American Sociological Review 27, 31--41 (1962)

7. Cho, D.S., Chu, W.: Determinants of Bargaing power in OEM Negotiations. Industrial Marketing Management 23, 343--355 (1994)

8. Pfeifer, P.E., Carraway, R.L.: Modeling customer relationships as Markov Chains. Journal of Interactive marketing, 14(2), 43--55 (2000)

9. Netzer, O., Lattin, J.M., Srinivasan, V.: A Hidden Markov Model of Customer Relationship Dynamics. Marketing Science 27(2), 185--204 (2008)

10. Madhusudanan P.V., Chandrasekharan M.P.: An absorbing Markov chain model for production systems with rework and scrapping. Computers \& Industrial Engineering 55: 695--706 (2008)

11. Xia J.C., Zeephongsekul P., Arrowsmith, C.: Modelling spatio-temporal movement of tourists using finite Markov chains. Mathematics and Computers in Simulation 79:154-$1553(2009)$

12. Llorca J., Delgado-Rodríguez M.: Competing risks analysis using Markov chains: impact of cerebrovascular and ischaemic heart disease in cáncer mortality. International Journal of Epidemiology 30:99--101 (2001)

13. Crossman R.J., Škulj D.: Imprecise Markov chains with absorption. International Journal of Approximate Reasoning 51:1085--1099 (2010) 